\title{
Business Models for Local 5G Micro Operators
}

\author{
Petri Ahokangas $^{1}$, Marja Matinmikko-Blue ${ }^{2}$, Seppo Yrjölä ${ }^{3}$, Veikko Seppänen ${ }^{1}$, \\ Heikki Hämmäinen ${ }^{4}$, Risto Jurva ${ }^{5}$, Matti Latva-aho ${ }^{2}$
}

\begin{abstract}
G will change the mobile communication business ecosystem by introducing location specific high-quality wireless networks that can by operated by different stakeholders. This development will change the traditional business models and ecosystem roles, as well as open the market for new local mobile network operators. These operators, such as recently introduced micro operators, can target specific customers in different vertical sectors with closed $5 \mathrm{G}$ networks, serve mobile network operator's (MNO) customers in high-demand areas on behalf of the MNO as a neutral host with open 5G networks, or mix different types of customers and offerings through various hybrid business models. This paper discusses business model options for local 5G micro operators, addressing also the different network deployment options. Three generic 5G business models and respective value ecosystems are presented: Vertical business model and ecosystem, Horizontal business model and ecosystem, and Oblique business model and ecosystem. Finally, the scalability, adaptability and sustainability of the business models and ecosystems are examined.
\end{abstract}

Keywords—business model;mobile network operator; spectrum sharing; micro operator; $5 G .^{1}$

\section{INTRODUCTION}

Today, $5 \mathrm{G}$ is considered to represent a disruptive technology for providing local context-specific connectivity and content services for various end users ranging from humans to machines. For this kind of highly localized and heterogeneous environments, the novel micro operator concept recently discussed in several papers [1]-[4] shows the promise as a means for speeding up the adoption of the $5 \mathrm{G}$ technology, while at the same time boosting local businesses and ecosystems to new growth areas. The two most important drivers of local and private networks with $5 \mathrm{G}$ are operations in higher carrier frequencies and the virtualization and componentization of the network infrastructure. The local micro operator concept has been discussed in [2] and [3] as an entity that combines connectivity with specific content services in spatially confined domains, being dependent on the availability of spectrum resources. In addition, appropriate

\footnotetext{
${ }^{1}$ Petri Ahokangas and Veikko Seppänen are with University of Oulu, Oulu Business School, Martti Ahtisaari Institute, Oulu, Finland.

${ }^{2}$ Marja Matinmikko-Blue and Matti Latva-aho are with University of Oulu, Centre for Wireless Communications (CWC), Oulu, Finland.

${ }^{3}$ Seppo Yrjölä is with Nokia, Oulu, Finland.

${ }^{4}$ Heikki Hämmäinen is with Aalto University, Department of

Communications and Networking, Helsinki, Finland.

${ }^{5}$ Risto Jurva is a free author.
}

business models are needed for micro operators to realize the innovation and growth potential embedded in this concept.

Future 5G business models have not yet been widely discussed in the research literature. The existing few examples focus on traditional mobile network operator (MNO) business models and discuss $5 \mathrm{G}$ in rather general terms. For example, authors in [5] introduced collaborative business models, [6] applied the brokerage business model to $5 \mathrm{G}$ businesses and [7] discussed a cloud-assisted business model. Beyond technicalities, all the three models mentioned can be seen to represent the two basic mobile operator business models; "bitpipe" and "differentiation" [8] [2]. They assume that mobile network operators are only to a limited degree interested to disrupt the dominant logic of the business, even if it opened up new growth opportunities. It is also worth noting that the localized nature of the $5 \mathrm{G}$ services was not seen a key characteristic in these studies. Some literature can be found to discuss, however, the antecedents to business models, the success factors [9] and the perspectives to be considered regarding $5 \mathrm{G}$ networks [10].

Four key business opportunities for locally confined micro operators have been presented in [4]: a) offering hosted local connectivity to all MNOs in specific locations, b) providing secure local networks for vertical-specific needs, c) providing locally tailored services and d) acting as a Mydata operator for various customers. The first of these seem appropriate in locations where it is neither feasible nor cost efficient that all MNOs would build their own infrastructure. The second could be a working solution in environments with highly specialized needs, such as production factories. Local tailoring of services may be needed when providing premium or personalized content, such as VR/AR services, or relevant context-specific or locally generated information. A key element in this opportunity is to manage and tailor the local infrastructure. Finally, Mydata operators might govern application or userspecific data and provide connectivity services on top of that, thus data forming the central aspect of the business opportunity.

The traditional MNO-centric way of delivering mobile/wireless services to end users locally is based on centralized infrastructure platform and control over the radio spectrum. Looking from the local perspective, especially in indoor environments and when using higher carrier frequencies, the new $5 \mathrm{G}$ ecosystem may bring in entirely new actors: facility owners, those who build and maintain the required infrastructures locally, and various content and equipment providers. This will open up new roles for mobile 
operators, mobile network vendors and local micro operators of various kinds as discussed in [3]-[4].

Building on the above discussion, this paper aims at identifying and discussing what kind of generic business models can be identified for emerging local $5 \mathrm{G}$ micro operators enabled by technological development and especially from the mobile business perspective. The key research questions that this paper seeks to answer are as follows:

1) What are the generic business models and ecosystem positions for micro operators to provide local services?

2) How may micro operators' business models differ from general MNO business models in local services?

The rest of the paper is organized as follows. First, 5G networks and new local operator approaches are introduced. Section III addresses the theoretical foundations of business models. Section IV presents the developed business models for local $5 \mathrm{G}$ micro operators. New $5 \mathrm{G}$ value ecosystems for the developed business models are derived in Section $\mathrm{V}$. Discussion is presented in Section VI, followed by conclusions and future directions in Section VII.

\section{5G MICRO OPERATORS}

Upcoming 5G networks are expected to offer reliable wireless connectivity to serve the versatile needs of different vertical sectors, as well as to provide even higher quality mobile broadband connections. Different 5G enabled services will have many and partially conflicting requirements in terms of quality and reliability, which must be met with the new system architecture and deployment models.

Provisioning of a high-quality connectivity infrastructure in specific locations such as schools, transport hubs, public service providers' units and enterprises has become an important societal objective as an enabler for new applications and services, see [11]. The context driven and location specific needs for wireless connectivity in different facilities have received increasing attention. Drastically new services that cannot even be predicted today may emerge. Different business cases for the deployment of $5 \mathrm{G}$ networks will arise in specific high-demand locations, including e.g. being a neutral host that provides connectivity services to traditional MNOs' customers in specific sites instead of all MNOs deploying their indoor networks separately, as discussed in [12]. Additionally, there is a growing interest towards local closed $5 \mathrm{G}$ networks that can be deployed and operated by different stakeholders to serve their own restricted sets of customers, for example in a factory environment.

Development of $5 \mathrm{G}$ networks aims at meeting increasingly stringent requirements for higher capacity, higher data rate, lower latency, massive device density, and reduced capital and operational costs. Moreover, 5G networks are planned to be deployed in a wide range of frequency bands, such as $3.5 \mathrm{GHz}$ and $26 / 28 \mathrm{GHz}$ in addition to the existing bands for mobile communications mainly below $3 \mathrm{GHz}$, which all have different deployment related characteristics.
From a technical viewpoint, 5G is expected to lead to [13]:

- a shift to ultra-dense small cell deployments,

- flexible network deployment and operation,

- multi-connectivity,

- $\quad$ security and privacy (data),

- dynamic traffic steering and resource management,

- intelligent use of network data,

- users participating in the storage,

- relaying,

- content delivery and computation within the network,

- coexistence of heterogeneous networks and local stand-alone $5 \mathrm{G}$ systems,

- the use of smart antennas to help in capacity and interference mitigation, and

- operations in higher (millimeter wave) frequencies.

On the network infrastructure side $5 \mathrm{G}$ is expected to take a leap from traditional network sharing between MNOs aimed at cost reductions, towards the on-demand multi-tenancy for hosting totally new services of specific customer segments. Network slicing functionality will be a critical new technical feature to enable multi-service and content-aware adaptation of the network to different applications, especially through dynamic creation of network slices on top of a common shared infrastructure [14]. Slices including both radio access networks and core network sides could span across the administrative domains of several stakeholders and be operated separately for the provisioning of services for specific customers.

Moreover, 5G developments have the potential to change the existing stakeholder roles and open up new roles in the future mobile communication business ecosystem. Several authors have proposed local high-quality $5 \mathrm{G}$ wireless networks to expand the traditional mobile broadband service offerings [4], [15]-[16]. In particular, the new micro operator role for locally deployed and operated small cell radio access networks is expected to emerge [2]-[4], to offer context-driven services and content with business models that will complement the known MNO offerings and business models.

\section{BUSINESS MODELS}

Business models have become a contemporary paradigm for designing, visualizing and communicating different business and service concepts and their implementations. Generally, business models help to answer the question what companies are offering to their customers in terms of products/services and value proposition, how and where they are planning to do that in practice, and why and how do they think they can do it profitably. A business model can be defined as a boundary-spanning unit of analysis [17] from the conceptual perspective or, practically, as a vehicle to exploit a business opportunity [18]. In a simplistic sense we may claim that a business model is an explanation of how an organization or an ecosystem of organizations carry out their business(es). Business models connect abstract strategies to their implementation on a practical level, and all business models should meet three key requirements; they should be scalable, adaptable and sustainable. A business model is thus related to three fundamental strategic business processes - exploration 
and exploitation of opportunities, co-creation and co-capture of value, and exploration and exploitation of competitive advantages [18]-[21].

All business model definitions address either how firms do business, how the way firms do business is interpreted by the (ecosystem) stakeholders, or how a business model could be represented by the means of formal conceptualizations [22]. In due course, ecosystems and business models within ecosystems are emerging as a new scope of strategy research. The content of a business model is reflected in its subcomponents. Yet, there is no unanimity between scholars with regard to the most essential business model components. For instance, a business model framework can include the customer interface, core strategy, strategic resources, and value network [23]. Also, one can distinguish between the design of the business transaction's content, structure and governance in the business model [18]. Moreover, the technology, market offering and network architecture can be considered the major constituent parts of a business model [24].

Noteworthy is that the traditional approach towards business model research focuses largely only on the supply side of value creation, not considering the demand side [22]. Authors in [25] suggest that when working together, business ecosystems allow companies to create such value that no single company could have created alone. However, business model conceptualizations are often created at the company level only, thus being less suited for analyzing the interdependent nature of businesses in ecosystem contexts. An ecosystemic business model incorporates the ideas of open innovation, expanding the boundaries of a company toward collaboration and coopetition, i.e., parallel collaboration and competition. A business model wheel concept introduced in [26] has widely been used in ecosystemic and future-oriented contexts [27]. The authors see the business opportunity at the heart of a business model, which comprises what (customers, offering, value proposition, differentiation), how (selling and marketing, delivery, key operations, basis of advantage), why (basis of pricing, way of charging, cost drivers, cost elements) and where (internal or external to a focal firm) elements (Fig 1.).

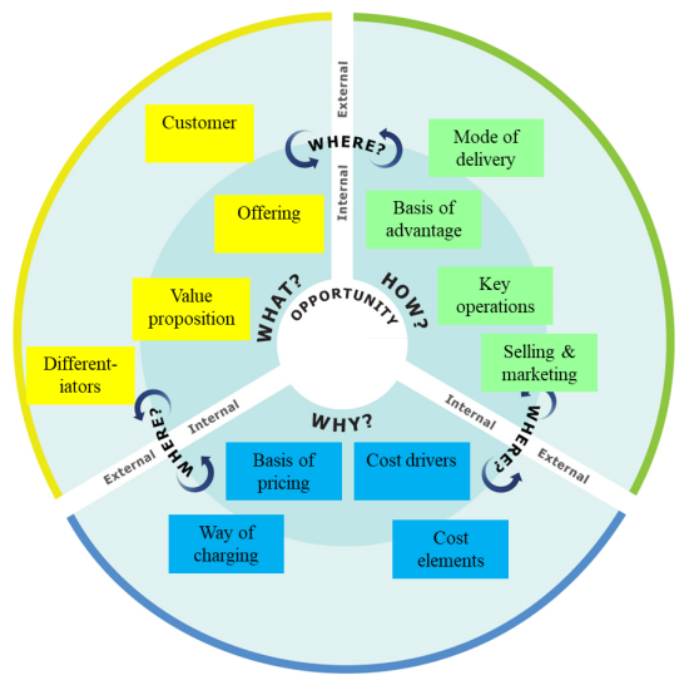

Fig. 1. The ecosystemic business model concept [26].
Attempts made to look at ecosystemic business models can be found in software, web/e-business, cloud, Internet-of-things (IoT), platform business, and wireless communications contexts. The well-known cloud/IoT classification of "as-aservice" business models from infrastructure-as-a-service to platform-as-a-service and software-as-a-service is widely used in various ICT domains, too. A typology presented in [28] suggests four types of business models for the Internet age to make the business model analysis easier and more structured. Each of the four types of business models have varying value propositions and revenue models: connection (e.g., wireless), content (e.g., data), context (e.g., search), and commerce (platforms). From the ecosystem perspective, the typology can be interpreted as a set of nested layers, where lower layer business models are required as enablers and value levers for the higher layers to exist [29].

A transformation of business models as well as entire industries can be seen to have happened [30] from vertical or horizontal linear to two-sided and networked. It can be pointed out that in two-sided business models also users create value. Taking a step further, with the emergence of platforms, [31] claimed that business models have started to turn "oblique", i.e., having a focus on value sharing through value co-creation and co-capture, while the traditional control-oriented vertical business models have aimed at controlling value creation and the horizontal business models controlling value capture. In other words, value sharing oriented ecosystems are emerging, focusing on co-creation of new services. In this co-creation, the key issue according to [32] is the openness of the business model. They see the openness of a business model starting from closed and extending toward open edge, open core and open source.

\section{The Developed 5G Business Models}

This section will present the developed $5 \mathrm{G}$ micro operator business models using the data collected from a series of workshops. Three generic micro-operator business models were identified in the analysis. These models were labeled as Vertical, Horizontal, and Oblique, building on the classification presented in [31]. In addition to these business models, we will analyze the ecosystems behind the micro operator business models and end the analysis by comparing the business models by their respective opportunities addressed, key value processes and core advantages, as well as their scalability, adaptability and sustainability. In the following, we will briefly introduce our research methodology and present the three generic business models identified.

\section{A. Research methodology}

In this research, we apply the anticipatory action learning (AAL) methodology for exploring complex and uncertain future businesses. The AAL method attempts to facilitate learning in a social system [33] through a democratic and collaborative visioning process that connects inquiry, anticipation and learning with action, assessment and decisionmaking [34] [35]. The method aims to make multiple levels of understanding merge openly and progressively during the process. AAL emphasizes the pluralistic reciprocal adjustment of research and reflects the exploration of alternative futures 
[36]. Both action research and action learning underline the necessity of experimenting, reflecting and learning form the exercises [37]. The participatory approach is practical for futures-oriented workshops to design innovative ideas through conceptual models, such as the business model. We strongly believe that the selected approach provides insights into plausible micro operator business models and ecosystem descriptions. We expect that the selected method will trigger business model innovation and lay a foundation for new business ecosystems.

The business model creation process that was utilized involved a series of facilitated face-to-face and online workshops that comprised representatives from major stakeholder groups identified within the micro operator ecosystem. The workshops involved designing business models by using the business model wheel as the conceptual model and practical tool. Cross-model reviews and comparisons by the workshop participants were used to deepen and clarify the designed business models and the ecosystem descriptions created based on the business models. The systematical evaluation and comparison of the business models was based on the dimensions presented in the theory section: the opportunity, value and advantage dimensions and the bases for scalability, adaptability and sustainability. All data and the presented arguments in the paper is based on workshop discussions.

\section{B. The Vertical business model}

When following the generic Vertical business model depicted in Fig. 2, the micro operator addresses the opportunity to provide tailored end-to-end services in restricted areas.

Typical customers in the Vertical model include industry automation verticals that may be segmented based e.g. on production type or industry, local utilities such as companies focusing on smart grid management, or local facilities from infrastructures to operational arenas. The Vertical business model builds on long-tail projects; each project is different, but scalability can be found by focusing on similarities across use cases. In other words, solution business opportunities emerge from tailored offerings that bear similarities. Machine-tomachine communications needed in IoT-enabled local industrial services is a good example of a use case in this model. Security and privacy, reliability and the management of local data may constitute important elements of such services, accompanied with smart means to analyze and make use of the gathered and communicated data.

What comes to the network life cycle, running the Vertical business model may comprise network planning, deployment, operation and maintenance, and differentiate in competition with its fast time-to-air and plug-and-play features.

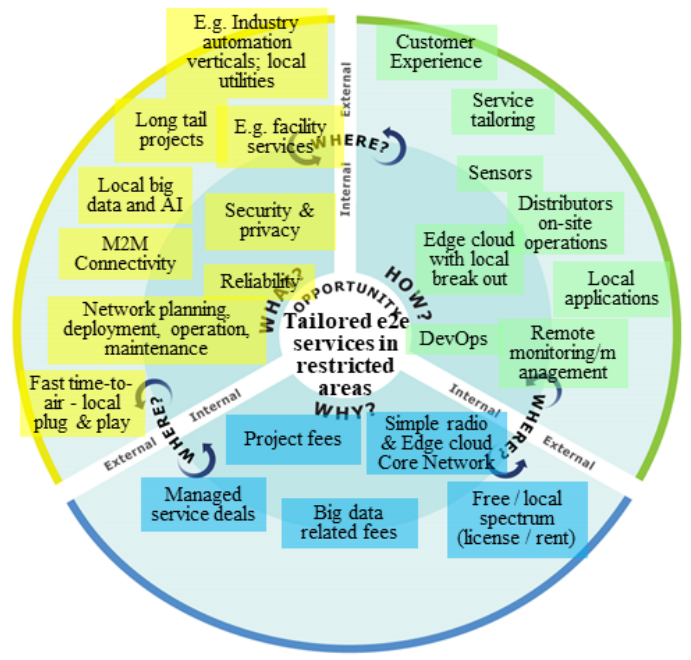

Fig. 2. The generic Vertical business model.

Sales in the Vertical business model is based on direct business-to-business selling, and delivery on the continuous DevOps/tailoring logic, where services are being flexibly developed or changed based on local customer needs to maintain customer experience and provisioned from the edge cloud with a local break-out. Typical services supported locally by the micro operator may include remote monitoring and management of operations or equipment and sensors, local applications run at the mobile edge, and various third-party operations ongoing in the facility/area of operation.

Charging is done in the Vertical business model to some degree through project fees, to cover the costs of building or taking in use the tailored network. However, pricing of the operation of the network may then be agreed through managed service deals. Additionally, it can also be based on the data sold to the stakeholders present in the facility/area of operation. Capital and operational expenses are tried to be minimized by using simple radios, the edge cloud, and the core network at the edge of the cloud. Local free or rented spectrum is utilized in communications. In other words, the cost of local connectivity is kept very low.

We identify four possible ecosystem stakeholders that could act as the micro operator with the Vertical business model: a factory or utility owner, an important factory machine vendor or a utility equipment provider, an $\mathrm{MNO}$, a network constructor, or such a public network provider as a city. Should an MNO be the micro operator, we face an interesting spectrum-related question: would the local (low cost) or MNO's own exclusive spectrum be used, when providing the service.

\section{The Horizontal business mdoel}

The Horizontal micro operator business, Fig. 3, model builds on very different grounds compared to the Vertical business model, namely the opportunity to provide local hosted connectivity for MNOs. In various public and restricted local places it might not be feasible that different MNOs would build their own networks. In such cases, e.g. at campuses, hospitals or shopping malls, it might be the case that a micro operator 
hosts MNOs' services locally to their customers and charges the MNOs for the service. The services hosted locally could comprise MNOs' standard services to their customers, but also bring MNOs additional capacity to them, ensure service continuity in an MNO's network, or provide improved network availability. Mass event organizers illustrate well such needs.

The selling logic for the micro operator in this case is direct business-to-business sales toward MNOs - typically in a white label format, where only the MNO's service brand would be visible to the end users. We expect that MNOs' motivation to buy the service from a micro operator is the guaranteed enduser experience. The micro operator could possibly provide the infrastructure as-a-service offering to the $\mathrm{MNO}$, and take care of the design, implementation, maintenance and also the removal of the network, if the need is temporary. Pricing could be based on capital and operational expenses of the service, managed through certain service level agreements and charged from the MNOs' utilizing the service. Spectrum could come from the MNOs served or be provided as a local free or lowcost/shared spectrum.

Again, we identify several potential stakeholders that could adopt the micro operator role: a network constructor, a facility owner, such a completely new actor as a capable third party, a cable or other operator or a joint venture established by a group of MNOs. Finally, various mass event organizers or site managers, and even construction companies might provide ad-hoc or other shorter-term $5 \mathrm{G}$ network operator services with the Horizontal business model.

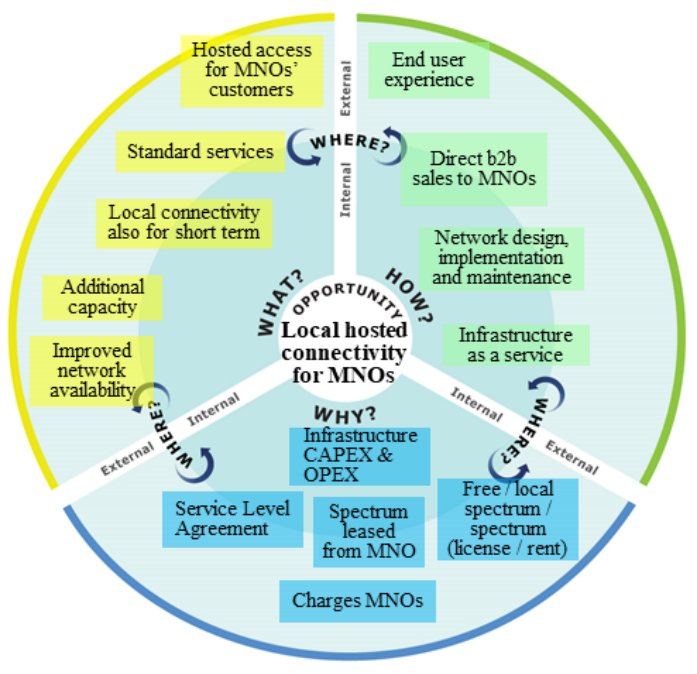

Fig. 3. The generic Horizontal business model.

\section{The Oblique business model}

The Oblique micro operator business model, Fig. 4, is based on an opportunity to provide mass-tailored end-to-end services to various segments.

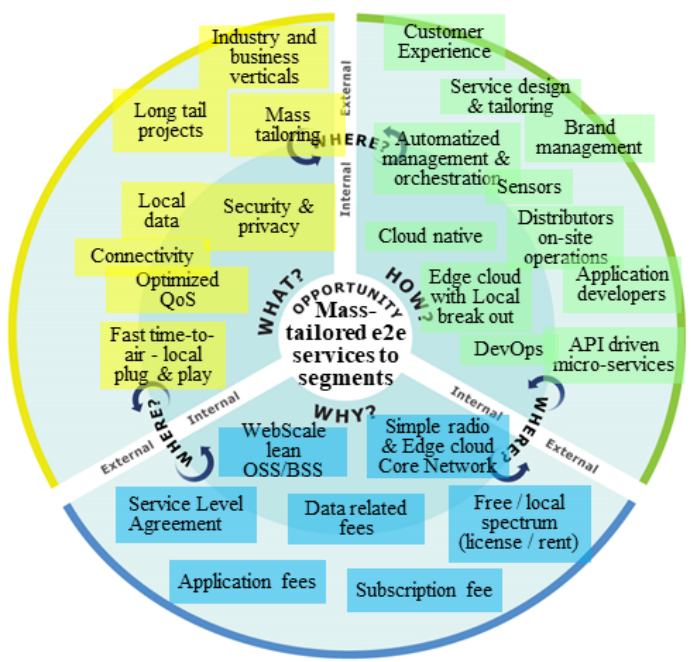

Fig. 4. The generic Oblique business model.

If in the Vertical business model scalability is based on similarities among use cases, in the Oblique model it is based on a platform that supports mass-tailoring. However, in this model, also, the micro operator offers connectivity with guaranteed security and privacy, local data and optimized quality of service. The platform should also support fast timeto-air and plug-and-play implementation across industry and business verticals. Because the platform approach supports bigger scalability compared to the Vertical model, with less need to take into consideration of the local specificities regarding e.g. the stakeholders present, MNOs and network infrastructure vendors could play the micro operator role locally. Both are capable to provide the edge cloud with a local break-out, run cloud native services and provide automated management and orchestration of local services.

What makes a difference and provides for transparency in the Oblique business model, compared to the Vertical model, are the micro operator's capabilities to build application programming driven micro services, and support application developers' activities and various on-site operations managed by third parties, i.e. to bring in customers' customers. The micro operator's brand creation and management is important in this. Webscale and lean operations support systems and business support systems (OSS/BSS), as well as simple radios with the edge cloud and the core network from the cloud could be utilized, bringing cost efficiency but providing also the possibility to monetize on data, application services and software. Finally, either free or local spectrum could be utilized locally, through licensing or renting.

\section{New 5G Value Ecosystems}

Business models need always to be calibrated to the respective business environment. A part of this environment consists of the surrounding ecosystem. The next step in the analysis involved mapping the ecosystem around the three designed business models for local micro operators. 


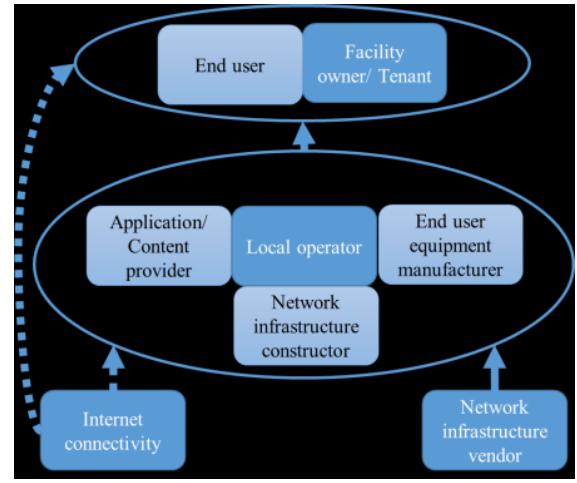

Fig. 5. The Vertical ecosystem.

The Vertical ecosystem is depicted in Fig. 5 (read the figure from bottom to top). It is characterized by the stakeholders' attempt to control value creation, delivery and capture. The local micro operator may try to exercise control by combining its local operator activities closely with application/content providers', end user equipment manufacturers', and network infrastructure constructors' activities through a project logic that may be extended with a long-term service contract.

Network infrastructure vendors and Internet connectivity providers can be utilized in a supplier mode, and the key customers are the facility (e.g., factory) owner under which the end users, whether machine or human, use the service. In the vertical ecosystem, the local micro operator role can be adopted by any stakeholder that possess advantages in the project that the facility owner needs, or in longer term in processing the data required in the facilities.

The Horizontal ecosystem is depicted in Fig. 6 (read the figure from left to right) and is very straightforward. The dominant player is the MNO, whose services the local micro operator delivers locally to the operator's customers. It may be stated that this ecosystem represents the logic with which mobile operators may extend their services to localized domains. The facility owner may collaborate closely with or even subsidy the local operator, but the network infrastructure vendor may collaborate even more closely with the local micro operator, as it can even adopt the local operator's role.

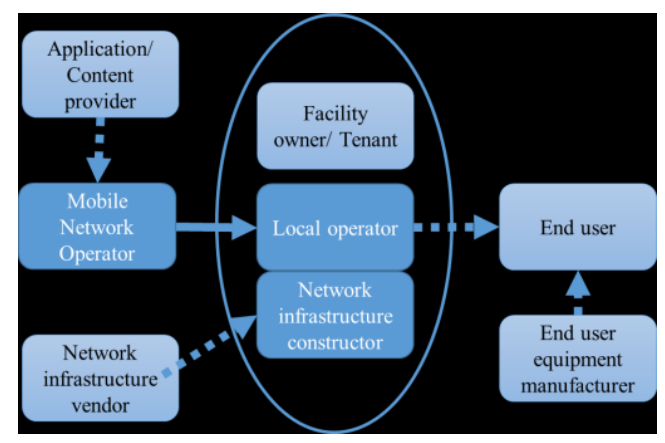

Fig. 6. The Horizontal ecosystem.
The Oblique ecosystem depicted in Fig. 7 (reading the figure can be started form anywhere) is the most complicated of the business ecosystem structures. To begin with, the network infrastructure constructor may help the local micro operator to build a platform that can be used to deliver services locally. At the same time, the local operator may closely collaborate with the application and content providers, whose services may be run on its infrastructure. From this platform the local micro operator may target several types of end-user or prosumer customer segments, while also selling their service to facility owners and third-party application and content providers that serve the end customers. It is noteworthy that the customer relationship between the local micro operator and the end user/prosumer segments may well be bidirectional, the same way as the relationship between the latter and third-party application and content providers. In this ecosystem, the mobile infrastructure vendors' role may increase due to core network slices that they could offer. Furthermore, management and orchestration of the virtualized network is required.

In the oblique ecosystem, any stakeholder willing to invest in designing, building, or maintaining local 5G infrastructures and services may adopt the local micro operator roles. This provides an opportunity for genuinely new players to enter the market.

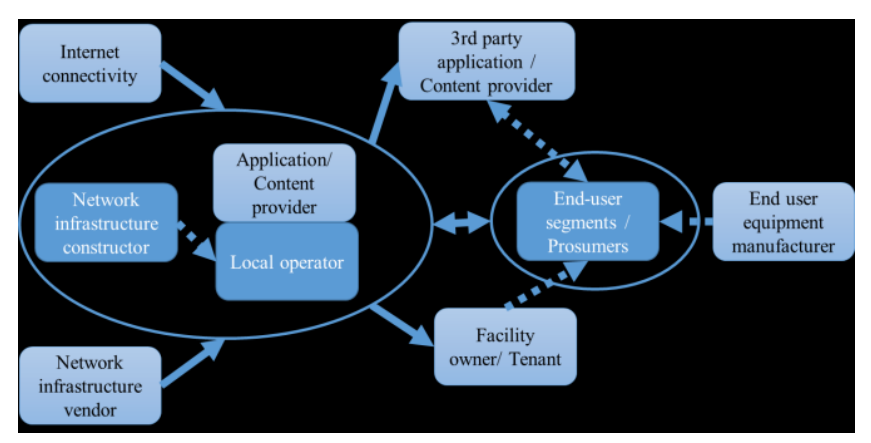

Fig. 7. The Oblique ecosystem.

\section{DISCUSSION}

To examine the designed business models and the respective ecosystem structures, we pay attention to opportunities exploited, sources of value, and advantages utilized. Next, we discuss the scalability, adaptability and sustainability of the business models in their ecosystem setting.

The key driver for any business model is the opportunity addressed. When compared across the three cases analyzed, the nature of the opportunity varies from customer specific, variable and changing needs in the vertical case via a generic but segmented need to continue enjoying existing MNOs' services beyond normal coverage in the horizontal case, to an opportunity to utilize mass tailoring when offering local services. Along with the change in the opportunity, also the type of the customer and the way how the local service is realized changes. A similar transformation may be observed regarding the way how value is created and delivered across the three cases - i.e., from projects to outsourcing and mass tailoring - and what kind of advantages are needed in 
providing local services - i.e., from technical competences to an existing customer base and a platform.

The presented ecosystem architectures indicate the logic and direction of potential scalability, adaptability, and sustainability in the respective business models. The Vertical business model do not indicate any substantial scalability potential over various customer cases, but may open up for existing project companies a potential to extend and upgrade their current offering, provided that they possess the capability to adapt to various customer needs and that the customer needs base is big enough to retain sustainability. As the Vertical business model follows the single or point product/service provider approach, scalability comes through generalization towards a solution provider, but possibly with neither explicit own and branded platform nor supply-side partner networks and systematic sharing of business assets among the micro operator's customer portfolio. Turn-key solutions may, however, provide for quite high-level profits, and earning may also be tied to subsequent use of the developed solutions. Upgrading and maintenance contracts could offer longer-term business opportunities and strengthen the micro operator's position in a particular vertical market.

The Horizontal business model's scalability potential rests on the competition among existing mobile operators and their subsequent need to increase cost-efficiency. In highly competitive environments the scalability potential of local micro operators increases, providing an opportunity for micro operators to build their own services. This may open new opportunities also to network constructors and mobile infrastructure vendors. Local micro operators do not need high adaptability regarding end customer services, but competences to design, build and manage local services in an efficient way become crucial.

The Horizontal business model rests ultimately on quite a straightforward supplementary offering to bigger MNOs in the same market and can be seen as a professional supplier or service provider business. The situation might be competitive among several MNOs or even towards a single MNO interested in investing to serve specific verticals, such as growing niche segments, or expanding its offerings through differentiation in its existing customer base. Compared to the MNOs', the local micro operator has not the power of directing the services of the end users in its possession but may still have special capabilities to bring them to the reach of the MNOs.

The scalability of the Oblique business model rests in the local micro operator's platform that enables mass tailoring better than those of the existing MNOs, especially when serving such specific needs as ones related to security, or the ability to run customer-specific local applications. To succeed, local micro operators need to combine platform flexibility with high adaptability to the local needs. The sustainability of the Oblique model may depend on the type and extent of local services requiring the $5 \mathrm{G}$ infrastructure. For example, VR/AR and high capacity AI based services may provide opportunities for genuinely new actors to start as local micro operators. An example of such could be an IT webscale company that utilizes network function virtualization and aims at reaching control over the network at its edges, thereby turning it from a telco market to an IT-centric market (cf. e.g. Amazon greengrass, Google, MS Azure IoT edge, Cisco).

The Oblique business model is obviously the most interesting one among the three and has the promise to include the best characteristics of the other two models. It is neither a plain local infrastructure-as-a-service for branded MNOs nor the heavily tailoring based and only gradually scalable business for many verticals. Instead, it represents a more elaborated platform approach, where the upstream supply side is opened to partners, but the downstream customer side kept in own hands. We have witnessed the general developments in digitalization, which are also supported by standardization and platformization via open interfaces, exactly to this direction. Whether the results will lead to "unfair" competition that "kills good existing businesses", or to a more effective use of underutilized resources, remains to be seen.

\section{CONCLUSIONS}

In this paper, we have explored alternative generic business models and the respective ecosystem constellations for local $5 \mathrm{G}$ micro operators including vertical, horizontal, and oblique business models. We have proposed a framework for approaching and examining ecosystemic business models and applied the approach through the anticipatory action learning methodology. The designed alternative business models for locally deployed $5 \mathrm{G}$ networks differ from the traditional MNOs' business models and are based on different business opportunities and value propositions. In addition, the life cycle focus of $5 \mathrm{G}$ networks - plan, build, operate - is different in the models. New ecosystem roles were identified, roles that might be adopted by several alternative but also completely new stakeholders. Also the cost drivers and elements, as well as pricing and charging vary remarkably among the business models. In sum, they represent very different types of businesses, as opposed to the presently dominant and rather homogeneous traditional MNO businesses.

It might not be exaggerated to claim that $5 \mathrm{G}$ will represent disruptive changes and create new growth opportunities in network operation businesses especially at the local level. Albeit, provided that the remaining regulatory challenges of local spectrum availability can be solved. Because $5 \mathrm{G}$ is still emerging as a technology, the actual development of business opportunities, business models and respective ecosystem alternatives remain to be seen. In particular, it is yet difficult to foresee which business model or what kind of market will start growing first and fastest, and if there will be some dominant stakeholders or a plethora of versatile micro operators around.

We acknowledge that regulation or spectrum allocations do not necessarily support the developments outlined in this paper everywhere yet. However, we have built our arguments assuming that necessary spectrum can be made available. Indeed, further research is needed to validate and evaluate the business models and ecosystems presented in this paper also from spectrum perspective, identify the key capabilities needed for running each of the models, open up and compare in details the value creation logic of each model, and consider regulatory barriers and enablers for each model in their ecosystemic contexts. 


\section{ACKNOWLEDGMENT}

This research has been financially supported by Business Finland in $\mathrm{uO} 5 \mathrm{G}$ project and Academy of Finland in 6Genesis Flagship (grant 318927). The authors would like to acknowledge the contributions of $\mathrm{uO} 5 \mathrm{G}$ project consortium.

\section{REFERENCES}

[1] M. G. Kibria, G. P. Villardi, K. Nguyen, W. S. Liao, K. Ishizu and F. Kojima, "Shared Spectrum Access Communications: A Neutral Host Micro Operator Approach," in IEEE Journal on Selected Areas in Communications, vol. 35, no. 8, pp. 1741-1753, Aug. 2017.

[2] P. Ahokangas, S. Moqaddamerad, M. Matinmikko, A. Abouzeid, I. Atkova, J. Gomes, M. Iivari, "Future micro operators business models in 5G'. The Business \& Management Review 7 (5), 143-149, 2016.

[3] M. Matinmikko, M. Latva-aho, P. Ahokangas, and V. Seppänen, "On regulations for 5G: Micro licensing for locally operated networks", Telecommunications Policy, (article in press) 2017.

[4] M. Matinmikko, M. Latva-aho, P. Ahokangas, S. Yrjölä, and T. Koivumäki, "Micro operators to boost local service delivery in $5 G$ ", Wireless Personal Communications, vol. 95, no. 1, pp. 69-82, Jul. 2017.

[5] J. Noll, M.M. Chowdhury, "5G: Service Continuity in Heterogeneous Environments," Wireless Personal Communications 57 (3), 413-429, 2011.

[6] T. Rasheed, A. Radwan, J. Rodriguez, J. Kibilda, R. Piesiewicz, C. Verikoukis, L.D. Gregorio, G. Gomes, T. Moreira, T., "Business models for cooperation," In Energy Efficient Smart Phones for $5 G$ Networks, A. Radwan, J. Rodriguez, Eds.,. Springer International Publishing, 241-267.

[7] N. Zhang, N. Cheng, A.T. Gamage, K. Zhang, J.W. Mark, X. Shen, "Cloud assisted HetNets toward 5G wireless networks," IEEE communications magazine 53 (6), 59-65, 2015.

[8] P. Ahokangas, M. Matinmikko, S. Yrjola, H. Okkonen. T. Casey," "Simple rules" for mobile network operators' strategic choices in future cognitive spectrum sharing networks," IEEE Wireless Communications 20 (2), 20-26, 2013.

[9] I. Neokosmidis, T, Rokkas, and D. Xydias "Roadmap to 5G success: Influencing factors and an innovative business model," In Internet of Things Business Models, Users, and Networks, 2017, pp. 1-8, IEEE, 2017.

[10] I.P. Chochliouros, A. Kostopoulos, A.S. Spiliopoulou, A. Dardamanis, I. Neokosmidis, T. Rokkas, L. Goratti, "Business and market perspectives in 5G networks." In Internet of Things Business Models, Users, and Networks, 2017, pp. 1-6. IEEE, 2017.

[11] European Commission. " $5 G$ for Europe: An action plan," Communication from the commission to the european parliament, the Council, the european economic and social committee and the committee of the regions. COM(2016)588 Final, 2016.

[12] P. Ahokangas, M. Matinmikko, S. Yrjölä, I. Atkova, "Disruptive revenue models for future micro operator driven mobile business ecosystem," Nordic Academy of Management Conference, Bodo, Norwaym 23-25 August, 2017.

[13] M. Agiwal, A. Roy, N. Saxena, "Next generation 5G wireless networks: A comprehensive survey," in IEEE Communications Surveys \& Tutorials, 18, 1617-1655, 2016.

[14] K. Samdanis, X. Costa-Perez, V. Sciancalepore, "From network sharing to multi-tenancy: The 5G network slice broker," IEEE Communications Magazine, 54, 32-39, 2016.
[15] M. D. P. Guirao, A. Wilzeck, A. Schmidt, K. Septinus and C. Thein, "Locally and temporary shared spectrum as opportunity for vertical sectors in 5G," IEEE Network, vol. 31, no. 6, pp. 24-31, 2017.

[16] J. Zander, "Beyond the ultra-dense barrier: Paradigm shifts on the road beyond $1000 \mathrm{x}$ wireless capacity", IEEE Wireless Communications, vol. 24, no. 3, pp. 96-102, Jan. 2017.

[17] C. Zott, R. Amit, L. Massa, "The business model: recent developments and future research," Journal of management 37 (4), 1019-1042, 2011.

[18] C. Zott, R Amit, "Business model design: an activity system perspective," Long range planning 43 (2), 216-226, 2010.

[19] H. Chesbrough, "Business model innovation: opportunities and barriers," Long range planning 43 (2), 354-363, 2010.

[20] D.J. Teece, "Business models, business strategy and innovation," Long range planning 43 (2), 172-194, 2010.

[21] P. Ahokangas, J. Myllykoski, "The practice of creating and transforming a business model," Journal of Business Models 2 (1), 6-18, 2014.

[22] L. Massa, C.L. Tucci, A. Afuah, "A critical assessment of business model research," Academy of Management Annals, 11(1), 73-104, 2017.

[23] G. Hamel, P. Ruben, Leading the revolution (Vol. 286). Boston, MA: Harvard Business School Press, 2000.

[24] K. Mason, M. Spring, "The sites and practices of business models," Industrial Marketing Management, 40(6), 1032-1041, 2011.

[25] R Adner, "Match your innovation strategy to your innovation ecosystem," Harvard business review, 84(4), 98, 2006.

[26] P. Ahokangas, M. Juntunen, J. Myllykoski, "Cloud computing and transformation of international e-business models",. In Building competences in dynamic environments, vol 7, Research in competencebased management, R. Sanchez, A. Heene Eds., London, Emerald Group, 2014. p. 3-28.

[27] P. Ahokangas, M. Matinmikko, S. Yrjola, M. Mustonen, H. Posti, E. Luttinen, A Kivimaki. "Business models for mobile network operators in Licensed Shared Access (LSA)." In Dynamic Spectrum Access Networks (DYSPAN), 2014 IEEE International Symposium on, pp. 263 270. IEEE, 2014.

[28] B.W. Wirtz, O. Schilke, S. Ullrich, "Strategic development of business models: implications of the Web 2.0 for creating value on the internet," Long range planning 43 (2), 272-290, 2010.

[29] S. Yrjölä, M. Matinmikko, P. Ahokangas, M. Mustonen, "Licensed shared access to spectrum," In Spectrum sharing in wireless networks. Fairness, efficiency and security, J.D. Matyjas., S. Kumar, F. Hu (Eds.), CRC Press, 2016. p. 139-164.

[30] S.P. Choudary, "Why Business Models Fail: Pipes vs. Platforms," Inovation Insights, Wired Magazine 2013. Retrieved January, 18, 2017.

[31] M. Iivari, P. Ahokangas, M. Komi, M. Tihinen, K. Valtanen, "Toward ecosystemic business models in the context of industrial internet," Journal of Business Models 4 (2), 42-59, 2016.

[32] R. Casadesus-Masanell, G. Llanes, "Mixed source," Management Science 57 (7), 1212-1230, 2011.

[33] T. Stevenson, "From vision into action," Futures 38 (6), 667-672, 2006.

[34] S. Inayatullah, "Questioning the future: Methods and tools for organizational and societal transformation," 2005.

[35] S. Inayatullah, "Anticipatory action learning: Theory and practice," Futures 38 (6), 656-666, 2006.

[36] T. Stevenson, "Anticipatory action learning: conversations about the future," Futures 34 (5), 417-425,2002.

[37] P. Reason, H. Bradbury, The SAGE Handbook of Action Research, (2nd ed.). Sage Publications Inc.: London, 2008. 